\title{
Can Reasons and Values Influence Action: How Might Intentional Agency Work Physiologically?
}

\author{
Raymond Noble ${ }^{1} \cdot$ Denis Noble ${ }^{2}$
}

Accepted: 18 September 2020 / Published online: 28 October 2020

(c) The Author(s) 2020

\begin{abstract}
In this paper, we demonstrate (1) how harnessing stochasticity can be the basis of creative agency; (2) that such harnessing can resolve the apparent conflict between reductionist (micro-level) accounts of behaviour and behaviour as the outcome of rational and value-driven (macro-level) decisions; (3) how neurophysiological processes can instantiate such behaviour; (4) The processes involved depend on three features of living organisms: (a) they are necessarily open systems; (b) micro-level systems therefore nest within higher-level systems; (c) causal interactions must occur across all the boundaries between the levels of organization. The higher levels constrain the dynamics of lower levels. The experimental evidence and theoretical arguments are shown to be consistent with previous research on the neuronal mechanisms of conscious choice, and with the interconnected multi-level processes by which organisms harness stochasticity, whether conscious or unconscious.
\end{abstract}

Keywords Harnessing stochasticity $\cdot$ Free choice $\cdot$ Free will $\cdot$ Intentionality $\cdot$ Agency Micro-level causation $\cdot$ Macro-level causation $\cdot$ Reductionism $\cdot$ Holism

\section{Introduction}

In recent articles (Noble 2017; Noble and Noble 2018; Noble and Noble 2017), we have shown how organisms can harness stochasticity in ways that serve functional needs. Organisms are not merely passive recipients of random variations at molecular and other levels. They can use stochasticity both to guide their behaviour creatively and to influence the directions in which they evolve. We have also shown that harnessing stochasticity can be the basis on which organisms make creative choices (Noble and Noble 2018). In that article, we drew attention to close parallels with the work of Karl Popper on evolution and free choice (Niemann 2014; Popper 1945; 1972; 1973; Popper and Eccles 1977).

Denis Noble

denis.noble@dpag.ox.ac.uk

Raymond Noble

r.noble@ucl.ac.uk

1 Institute for Women's Health, University College London, London WC1E 6AU, UK

2 Department of Physiology, Anatomy and Genetics, University of Oxford, Oxford OX1 3PT, UK 
In this paper, we will explore whether harnessing stochasticity could be the basis of resolving a long-standing philosophical problem: the tension between what are thought to be standard empirical (usually mechanistic) scientific accounts of organism behaviour and views of life that regard behaviour as the outcome of rational and value-driven decisions. We will refer to the first as micro-level and the second as macro-level explanations.

On the macro-level view, organisms can act rationally in the sense that we can give (in the case of humans) or we can see (in the case of other organisms) good social reasons why they chose to act in the way they do. The micro-level view is often taken by empirical scientists to be that those reasons were not the cause of the behaviour. On that account, the behaviour was entirely accountable by observing the physical processes involved. This is the basis of the reductionist view of biology. Could both accounts be somehow correct, or must there be a, perhaps unresolvable, tension between them?

On any micro-level view, the tension is evident. If determinate molecular and other physical forces wholly and always determine the actions of organisms, and if there is causal closure, then how could a macro-level explanation involving rational or value-driven action possibly be correct? The philosopher Jaegwon Kim, for example, argues persuasively that if there is complete causal closure at the microphysical (e.g. molecular) level then there is no room for additional causation from macro-levels (Kim 2000). The feelings and thoughts we have as humans must then be an illusion (Heisenberg 2009; Midgley 2014), and to work it has to be assumed to be a very strong illusion. That is precisely what incompatibilist evolutionary biologists suppose:

"The illusion of agency is so powerful that even strong incompatibilists like myself will always act as if we had choices, even though we know that we don't. We have no choice in this matter. But we can at least ponder why evolution might have bequeathed us such a powerful illusion."(Coyne 2014)

Intention and will can be admitted to exist (how could we possibly deny that they exist as experienced by us?), but on this view they are not causally involved.

In this article we challenge two assumptions in the purely micro-level view. The first is the possibility of causal closure in living organisms, meaning that there is no room for any other influence, particularly that of intention; the second is the assumption that any stochasticity involved cannot itself be functional.

The assumption of causal closure cannot be true of living organisms since they are necessarily open systems (for recent accounts of organisms as open systems, see (Capra and Luisi 2014; Noble 2016). In this paper, we will explain the consequences for causal explanation at both micro- and macro-levels (see Sect. $4 \mathrm{~g}$ ).

\section{The Origins of Stochasticity}

Thermal and quantal noise exists at the molecular, atomic and subatomic levels (Del Santo and Gisin 2019), while the causes that organisms encounter in their interactions with other organisms and with their environments include many forms of chance occurrence. However, these facts, by themselves, are insufficient to resolve the tension between macro- and micro-level explanations. There are degrees of uncertainty. Intention and will are not claimed to be just chance. They are claimed to be causally influential on the macrolevel view. Thus, mimicking organisms by simply introducing stochastic equations into otherwise determinate algorithms in artificial intelligence systems can generate novel and 
unpredictable behaviour but does not create what we call agency (Noble and Noble 2019), i.e. when organisms make decisions or have an intention to act. Introducing unharnessed stochasticity is merely an additional reductive process.

Furthermore, regarding biological systems as determinate, but requiring the addition of a creative element, is to misunderstand that biological systems are organisationally creative. We will also explain the kind of causality involved. For that, we need to demonstrate a rationalising biological process that enables organisms to make choices predicated on it.

We will outline physiological processes that can enable rational and value-driven decisions in behaviour to be causally influential. The evidence is that harnessing stochasticity in the way in which we described this process in (Noble and Noble 2018) could be the basis of agency and therefore of free, rational action. It is the organism that is doing what it is doing with the intention to achieve an objective. In a previous article (Noble et al. 2019) we took this approach further to characterise more precisely how downward (i.e. macrolevel) causation is effective physiologically. We gave detailed examples where choices of life-style influence molecular events that control our genes.

We will also show that the processes we outline could be compatible with recent philosophical work on the nature of free action, specifically the account presented in the work of Christian List (2019)—see Sect. 6.

\section{Causation}

Some of the arguments in this paper turn on the meaning of causation. Not all causes are of the same type. We use the categories of causation derived from Aristotle. A version of these categories of use in multi-level biology can be found in Noble (2016, 176-181), which includes any factors whose alteration would result in changes in behaviour. Specifically we distinguish between the dynamics of a system and the conditions under which those dynamics play out. Those conditions can then be seen as constraining the dynamics, just as the dimensions and elasticity of the container of a gas determine the pressure and temperature generated by the dynamics of the molecular movements. In the biology of conscious organisms, constraints will necessarily include social/cultural factors. Some of those will be logical ones, including what in ordinary language we would refer to as the reasons for an organism's behaviour. This issue is analysed further in Sect. 7 of the paper.

\section{The Argument}

\subsection{Why Do We Need a Macro-Level Account?}

The tension between micro-level and macro-level accounts might be 'solved' by claiming that they are merely different viewpoints. The micro-level account is then regarded as the (only?) scientific one, while the macro-level account is the (necessary for ordinary social interactions?) philosophical one. However, this solution is unsatisfactory not only because it fails to explain how rational thought can influence behaviour, and how this can be a basis of 'free' choice, but also because it matters to society whether we believe we are living under a strong illusion (Vohs and Schooler 2008). Socially it begs the question what illusion we are living with. If rational thought is not influential, why then do we also need the 
macro-level (common sense) explanation? Why is it necessary? And why should we even strive to act according to what we feel and want?

The answer is that, as Coyne acknowledges (we "always act as if we had choices"), we need the macro-level account because, in practice, we cannot manage without it. In the real world, we need to treat other people and many other organisms as intentional beings with real rational choices to make. When we ask someone else "why did you do that?" we do not usually expect the answer "my brain/genes, or whatever, made me do it." (Murphy and Brown 2007; Lim 2008). We expect a rational explanation. That is so even if it may be a rationalised explanation. Whether we or the other organism believe the explanation or not, we need it in order to continue a rational interaction and often to make choices. Interactions between predator and prey often involve anticipating the behaviour of each other. This is also true of human interactions since our intentions are predicated on the intentions of others. In this sense the 'illusion' of intention is powerful because we act upon it. Merely calling intentionality an illusion does not solve the problem; it merely parks it.

Such interaction may also change the other person's perception of their behaviour, e.g. by giving the reasons why their rationalisation, or reasoning, cannot be correct, and this interaction influences what they do. We do this all the time in argumentation, and in legal contexts, yet none of it appears to make sense in a purely micro-level explanation (Ellis 2016; Midgley 2014).

In fact, on the micro-level explanation, someone's reasons for the action they took would never be the 'real' causes. At best they might be shorthand for the micro-level account. This is the approach taken by some evolutionary biologists. As an example, Maynard Smith $(1998,213)$ wrote:

I am prepared to think as loosely as necessary to give me an idea when I'm confronted with a new biological problem.....But when I've got an idea, I want to be able to write down the equations and show that the idea works.

Purposive accounts of behaviour or function in organisms are then temporary shorthand for mechanistic accounts in terms of gene variations and natural selection. It matters to decide whether this 'shorthand' view is correct. We will show in Sect. 4(g) that it matters what kind of mathematics is used.

\subsection{Physiological Evidence for Micro-Level Causal Efficacy of Rational Choice}

Recent physiological research shows that it is possible to measure micro-level effects contingent upon macro-level decision-making. As an example, consider the case where an athlete chooses, as his life-style, to train hard and regularly. This decision subjects his body to many physiological challenges as he deliberately pushes these physiological processes to their limits. Many of the decisions he or she makes will be influenced by reading articles on fitness, or interactions and advice from his trainer, and by observing others. These decisions are made at a social level and are logically based on an understanding of physiology and training. Any particular form of exercise is performed to produce a given result. We have discussed this case in detail in a previous article (Noble et al. 2019). What happens? One thing that happens over time is that the decision and training as an athlete cause RNA changes that enable the production of more of the right muscle proteins that enhance his athletic ability (Bathgate et al. 2018). Indeed, much more than that happens. His cardiovascular fitness improves, he gets better at making decisions, when to run faster and how to pace himself. He may also alter his diet and make choices about what to eat and 
when. The rational process in making these decisions therefore had a profound effect at both macro- and micro-levels. It seems implausible to argue that all of these changes could be determined purely by the evolutionary process since they occur during the lifetime of the individual.

Gene-determinists might nevertheless argue that the athlete just had the right genes to enable him to be a successful athlete (Plomin 2018), whether or not he uses them. That must be true, of course, at least in an enabling sense (Epstein 2014). However, so did his identical twin brother who did not become an athlete (Bathgate et al. 2018). Identical twins are born with the same genome. The difference between them is in no small measure the decision by the athletic brother to exercise regularly, to change his lifestyle. Genes were a necessary condition, but they do not and obviously cannot fully explain the difference between identical twin brothers.

The difference between the identical twins might be explained in part by their different experiences in the womb. Being identical in a genome sense does not mean they developed identically in the womb. Blood supply is often not evenly distributed between twins, so their nutrient experience may be different, leading to a difference in metabolic strategy and also possibly in muscle type. They may also have experienced different levels of oestrogen and testosterone in the womb. We should be aware of this alternative explanation for differences between twins since those prenatal influences might predispose one twin rather than the other in a particular direction. But the fact remains that the RNA changes will occur only if and when the life-style choice is made and maintained. Anything that prevents that from happening would also prevent the micro-level RNA and similar changes from happening.

Physiologists are identifying many other molecular epigenetic changes consequent upon life-style social choices that change how gene expression levels are varied. These experiments show that even on the micro-level approach of measuring low-level molecular changes, rational choices have causal effects. Thus, they show that the issue between micro-level and macro-level accounts is not a purely conceptual problem. It is an empirical matter that it can be and is resolved in the sense that a rational choice influenced the physical events.

\subsection{Evidence from Observations of Non-Human Animals}

Other organisms are also capable of this kind of agency. When chimpanzees or bonobos use body language and signs, such as hand gestures and facial expression, to communicate with each other they do so anticipating the possible responses of each other. The precise meaning of a sign is dependent on the contextual logic. They act with reason and anticipate with reason. Language is like a tool to convey anticipatory states (Byrne et al. 2017; Graham et al. 2017, 2018; Hobsiter and Byrne 2014).

As a further example, when a chimpanzee uses a stone to crack-open a nut, it is not a hard-wired biogenically determined event. It is a creative choice. Furthermore, chimpanzees choose stones that are better suited for cracking nuts, and in that sense therefore the behaviour is rational (Whiten 2017). Chimpanzees learn this by observing others and by their experience, solving a problem by using tools. Tools are purposeful because of the agency of their creators. The question is not whether but how biological systems achieve this creativity.

If there is perceived to be a tension between micro-level and macro-level explanations, then we contend that physiological processes themselves, as open systems, show that the 
tension is in principle resolved, and these physiological findings can demonstrate that. The question remaining is how is this possible? What kind of physiological processes could account for it?

\subsection{The Proposed Resolution}

We will now outline how such physiological changes can, at the same time, have identifiable molecular biological processes by which they occur and also satisfy the criterion of rational choice. To achieve that we need to outline a physiological process that would explain why we are not necessarily able to make predictions in advance but the choice will appear rational in retrospect. The philosopher John Lucas expressed this requirement in his book The Freedom of the Will:

For the reasons which "determine" a composer to add a particular bar to his composition are parts of some sort of rational explanation, not a regularity one; they do not enable us to make predictions in advance, but only to see how right it was ex post facto. It is the mark of creative genius that it is original and unpredictable; although after it has manifested itself, its rationale is manifest also. (Lucas 1970)

Using the example of creative genius is simply a way of highlighting this difference between what we can say in advance and the rationale we can give afterwards. The difference is to varying degrees characteristic of all rational behaviour.

The reason why this conjunction seems impossible is that molecular processes, in themselves, cannot have values and meaning of the kind that is necessary to satisfy the criterion of rationality. A micro-level approach does not find the rational self. The idea that genes make us selfish, or that the overriding purpose of our behaviour is to preserve genes in a gene pool, is to impart purpose (albeit metaphorically) to an inanimate bit of the microlevel system yet deny it to a macro-level organism. DNA, RNA or protein sequences, and the lipids and metabolites with which they interact in functional biological networks have no meaning outside the context of a living organism that can give them meaning.

This point about gene sequences is valid in the same way in which alphabetic sequences in a language have no meaning outside the context of the language speakers who choose to give them meaning. Thus, the sequence B U T has completely different meanings in English and French. Similarly, gene sequences involved in the development of arms and legs have entirely different functional instantiations in organisms without arms or legs. A single gene (per in the fruit fly) can be involved in functions as different as circadian rhythm, long-term memory, cancer development, and courtship behaviour (Foster and Kreitzman 2004; Sakai et al. 2004). Genes are not by themselves causal. They can have no property of motivation or agency.

Furthermore, in organisms, the molecular sequences do not have meaning in isolation. Gene ontology (the giving of names to genes based on their functional involvement in the functions of the organism) may give the popular impression of 'genes for this' and 'genes for that'. What the genome-wide association studies (GWAS) have shown is that this is far from reality. Many DNA and RNA sequences are involved in every function of the body. Some GWAS scientists even favour the omnigenic hypothesis (Boyle et al. 2017), which surmises that all genes are involved in one way or another in all functions. That is another way of expressing the fact that all molecular sequences have no meaning outside the context of the complete organism. 
These are important scientific discoveries. However, the central conceptual tension remains. If there was only one, determinate, molecular level response to a given challenging situation for the organism, the chances of it also happening to be the one that at the molecular level instantiates the rational decision at a macro-level would be so small as effectively to be zero. We base our claim on the fact that this is not how organisms react to challenging situations. We need to abandon the twin ideas of linear-sequenced causality and privileged level causality. As we showed in (Noble et al. 2019), the forms of causation that act across boundaries necessarily act simultaneously, and they are necessarily different forms of causation.

We will first show what organisms can do in a non-neuronal (or unconscious) process and then explain how similar processes of harnessing stochasticity must occur in nervous, including conscious, processes.

In both cases, organisms can harness stochasticity in ways that generate rational (i.e. guided) responses to environmental challenges. The empirical foundation was laid out in (Noble 2017) and applied to agency driven processes in (Noble and Noble 2017; Noble and Noble 2018). Organisms have demonstrably evolved guided random mutation and other molecular mechanisms that can respond rapidly and correctly to environmental challenges. These processes allow organisms and populations to harness stochasticity to evolve solutions to such challenges relatively fast compared to the accumulation of non-harnessed chance variations. It is the harnessing of stochasticity in guided responses to environmental challenges that achieves what blind chance alone could not possibly do. Ellis (2016: 163-168) refers to such processes as Adaptive Selection of Outcomes. Hoffmann refers to it as the process of extracting order from chaos in his book Life's Ratchet (Hoffmann 2012). He correctly identifies many of the molecular mechanisms that enable this extraction to occur, and concludes that

reductionism is essential if we want to understand life. Without it, scientists would have long ago stopped looking at smaller and smaller scales and would have missed the marvels of molecular machinery. At the same time, molecular machines don't explain everything. Scientists must still answer the question of how these machines interact. The ultimate goal is always to explain the totality of life's processes..... reductionism and holism are two sides of the same coin. (Hoffmann 2012, 238)

Bronfman et al. (2016) refer to

hierarchical predictive coding theory, according to which one of the organism's biggest challenges is to infer the (hidden) world-causes that give rise to the (observable) sensory signals the animal receives.

This point is relevant to ours about the anticipatory behaviour of organisms (Sect. 4(f)).

\subsection{Example of Guided Reaction to New Environmental Stress: The Immune System}

The immune system achieves such guided responses throughout the life of an organism. The mutation rate in the variable part of the genome that forms the template for an immunoglobulin protein can be accelerated by many orders of magnitude in response to a new antigen challenge. So far as is known, these mutations occur stochastically, and what is modified is the speed at which they occur. However, the location in the genome is certainly not a matter of chance. The functionality, in this case, lies precisely in targeting the relevant part of the genome. The arrival of the antigen itself activates the hypermutation 
process, and the binding to a successful antibody triggers the proliferation of those cells that make it. Thus, the system targets the specific antigen.

What this process achieves is that all the other sequences in the DNA array forming a template for the immunoglobulin protein are held sufficiently constant to maintain functionality. Even more remarkably, all the functionality in the rest of the genome is also maintained. Considering the vast size of the entire genome, this is pin-point targeting requiring highly specific feedback processes to be successful.

By holding correct parts of the immunoglobulin sequence constant, the system finely tunes the rapid mutation to only a tiny part of the entire genome. Such tuning is one way in which organisms can dynamically respond to environmental change (Noble 2018). Another way is that their biological networks buffer the organism from the majority of molecular changes at the genetic level. The robustness of the networks acts like a cloud overlying the DNA (Noble and Noble 2017) so that, under favourable environmental conditions, as much as $80 \%$ of the genome changes have negligible effects (Hillenmeyer et al. 2008).

The targeted process in the immune system has been known and intensely studied for many years (Odegard and Schatz 2006; Shapiro 2011). The molecular action has been identified as abolishing or reducing the DNA copying error-correction process (Saribasak and Gearhart 2012). So, how did many people not realise that it is a physiologically guided process? The answer is that the guidance does not lie at the genome level. At the genome level, the process appears blind. It depends on purely stochastic mutation. The functionality guiding or targetting the process lies in the system as a whole. The system harnesses the stochasticity. The immune system is the paradigm example of this harnessing.

The system includes: (a) sensing the environmental challenge, i.e. the antigen invasion, (b) transmitting this signal to the nuclei of immune system cells to trigger hyper-mutation in just a tiny fraction of the genome, (c) then sensing of the correctness or otherwise of the outcome, followed by the "reproduce or die" signal: cells that do not produce an antibody to the antigen do not reproduce. At this stage, natural selection occurs amongst the population of immune system cells. Thus, this is a complete, finely-tuned physiological feedback and guided search system rapidly generating an acquired characteristic in response to an environmental challenge, and inherited through the surviving population of cells. By all the usual criteria this is a teleological, i.e. goal-directed, process.

The process does not have to be perfect. Not all keys have to be a perfect fit to open a lock. The system feels its way forward, harnessing stochasticity to create novelty while using targeted preservation of what already works. The targeted preservation is what gives the system its purpose: to maintain its integrity. It uses stochasticity to change what it must change, precisely because that is the part that does not work or does not work well enough.

It is crucial moreover to see that the goal, the directionality, exists within the organisms and their populations. The goals of organisms and populations of organisms have developed during the evolutionary process. They are harnessing the capacity to change to meet environmental or psycho-social challenges.

\subsection{Harnessing Stochasticity Within the Nervous System}

In a previous article on similar processes in the nervous system (Noble and Noble 2018), we represented the process as follows, where we divide what happens into steps only for clarity. There are significant analogies with, but also substantive differences from, processes in the immune system, which harnesses stochasticity to create antibodies to new challenges. 
1. Influences from the environment and the organism's history may present a particular problem to the organism-for example, gaining access to a food supply or defending itself from a potential predator, or building a nest. We conjecture that such a problem can be viewed as a puzzle for which the organism needs a solution. The challenge facing the organism is in finding an answer to the puzzle. The puzzle is complex and multidimensional in space and time.

Behaviour is an ongoing dynamic process and is iterative and reiterative in solution and execution. Nor is the puzzle itself static, and the response is equally fluid or elastic, requiring recalculation and reassessment in finding a solution, for example in a predator stalking its prey. Once we engage a solution, more decisions are needed in addition to the original one. Problems do not present themselves in isolation, although we might dissect them as if they did. Thus, decisions of some kind are necessary even with routine outputs. The decision process is not a linear sequence, and the processing of action is continually assessed and adjusted; in a predator-prey situation where each party is continuously assessing, or anticipating the intent of the other.

2. Part of the process of choice is that organisms consider experience and memory of what may work as a solution. We hypothesise that the harnessing of stochasticity occurs continuously. Thus the organism is continually activating stochastic processes within and without itself so generating ongoing creative solutions. The stochastic options available may be extensive and at various levels (Atmanspacher and Rotter 2011; Brembs and Heisenberg 2018; Burns 1968; Hille 1992; Tchaptchet et al. 2015).

For example, the solution for a chimpanzee in getting termites would be to push a stick into the nest to get them. Both the use of the stick and choosing to hone it or modify it to better effect are biologically and socially creative and rational solutions in action. The behaviour can be understood both in terms of the biology and the situational logic. If we see a chimpanzee honing a stick, we (and other chimpanzees) might reasonably anticipate that it is going to use it to get termites.

Where are these stochastic processes located in the case of the nervous system? The answer is at all levels. Stochasticity is ubiquitous in biological systems.

At the cellular level, all life depends on a mostly stochastic process in generating a membrane potential. The generation of that potential depends on the concentration gradients of positive and negative ions driving flux across the membrane.

Control of these random fluxes is by changes in membrane permeability-opening and closing of specific ion channels. By opening ion channels in the membrane, nerve cells harness this stochasticity to generate discrete electrical signals, action potentials. Thus, neural processes are extensively stochastic — at all functional levels, from the opening and closing of ion channels via action potential generation, spontaneously or through synaptic transmission in neuronal networks, up to cognitive functions including decision making. All involve an ebb and flow of inhibitory and excitatory processes in complex networks of neurones. This balance is so even for the processing of first-order sensory inputs involving both localised and descending modulation of sensory processing in anticipation of a stimulus (Noble and Short 1989; Noble and Riddell 1989). To quote from the second of those papers:

Nevertheless, it is clear from the present study that the PSDC [postsynaptic dorsal column neurones] system is amongst those somaesthetic pathways under the influ- 
ence of descending mechanisms which, whatever their precise physiological function, have the potential to modify and regulate the transmission of cutaneous sensory messages. (Noble and Riddell 1989, 181).

Thus, stochasticity is harnessed in the functional organisation of living systems composed of a manifold of nonlinear feedback loops that often are adjusted to operate in the neighbourhood of bifurcations where what happens can significantly depend on random influences, e.g. whether an ion channel is opened or remains closed or whether action potentials arise or not.

3. Potential solutions using these stochastic processes are continuously assessed as solutions to the puzzle. While a particular problem might be present at a given moment, the processes involved are in continuous engagement in anticipation of such problems forming part of perception and awareness.

4. The capacity to abstract the problem and potential solution may depend on the complexity of the organisation in a given species. Thus, in humans, we can envisage the potential solution and rehearse it before the behavioural output. Other species may also do so.

This process helps to explain the apparent paradox that we referred to earlier regarding the predictability or otherwise of what we call a free choice, and it ensures that, in retrospect, the choice may be what in the case of humans we call rational. There may be a greater or lesser degree of conformity to possible reasons why it was made. So the choice is both rational and creative.

We can now answer the earlier question in Sect. 4(d): how physiological changes can at the same time have identifiable molecular biological processes by which they occur and also correspond to rational choice at a macro-level. By harnessing stochasticity, lower-level attempts at a solution might occur, some of which correspond to an appropriate rational choice. The analogy with the immune system is now apparent in the harnessing of stochasticity, but the nervous system processes are different in many other respects.

\subsection{Organisms as Open Systems}

Organisms are open systems at both micro- and macro-levels. Thus, there are no hard boundaries to causation between levels; or as Capra and Luisi (2014) express it:

In nature, there is no 'above' or 'below', and there are no hierarchies. There are only networks nesting within other networks.

'Nesting' is the key. Each level of function meshes with and so interacts with other levels (Noble et al. 2019). Molecular networks nest within cells, which in turn nest within cellular (tissue) networks, within organ networks, and whole organism networks, then within their social networks, and also in interaction with other species (ecology). This meshwork is what we mean by open systems - while there may be causal distance, there is no causal isolation. Thus, boundaries are not merely structural; they are also functional. Biological systems are not closed mechanical units. What happens at a social level of organisation influences what happens at the level of organs, tissues, cells and ultimately, molecules. Perceptions of the world, and each other, are influenced by culture and social interaction; it influences the development of our brains and our thoughts. Spinning the stochastic wheel occurs at all levels, allowing biological systems to be creative. 
Where we differ from Capra and Luisi is on the question of hierarchy. In systems with agency, there is a hierarchy of sorts. The decisions of the agent, the organism, influence function at other levels, even as function at other levels forms the integral being of the agent. The level at which agency can exist constrains all the other levels. However, an agent depends on the capacity to act, which in turn is constrained by functionality. In that sense, there is no privileged level of causality; all levels engage in the process of agency, of making decisions and acting on them. What is the agent, but the organism, or the social entity? Being aware or conscious of our agency is also a functional level. It is not merely an 'illusion' but a potent level of organisation. Furthermore, the expression of our thoughts, our ideas, framing them in language and social interaction is a functional boundary.

This insight into the nature of open systems derives from a mathematical approach to the interactions across boundaries between different organisational levels, formulated as the principle of biological relativity (Noble 2012). It is important to note that the distinction between dynamics and constraints is evident in differential equation models but not in "static" algebraic models often used in evolutionary biology, for example in models of kin selection. When challenged by Stephen Rose on whether his work on algebraic models of kin selection and altruism (Smith and John 1964) could have political, philosophical and economic consequences, the evolutionary biologist John Maynard Smith famously quipped "what would he have me do? Fiddle the algebra?" (Smith 1979). Significantly, had Maynard Smith been using dynamic differential equation models he might have seen that constraints from macro-levels require no "fiddling" of the dynamic equations. Static models leave little room for the social dynamics of altruism and concepts of good and bad behaviour or thought, or individual agency. Static mathematics describes a system requiring stasis or equilibrium, or 'maintaining genes in a gene pool' and becomes the overriding goal of the system. In that case, the mathematics governs us rather than the other way round. However, the form of mathematics is a choice we have made. Evolutionary biology and its contributions to the debates on agency might look very different today had it not been so profoundly influenced by static (steady-state) mathematical models. So also would the philosophical debates on agency and free action. Organisms, as open systems, are never in a static equilibrium state.

\section{Relevant Previous Physiological Work}

Our proposed reconciliation between micro- and macro-level accounts requires that nervous systems are capable of generating different possible courses of actions of which the organism may be conscious. This capacity is what empirical studies of conscious awareness propose. Crick and Koch (2003; Koch 2012), for example, specifically talk of the processes of attention and neural selection processes in their work on the physiology of consciousness. We dissent from their reductionist approach to consciousness and in particular from the tendency in reductionist accounts to create a form of mechanistic dualism by reifying a specific bit of the process, a 'seat of consciousness' somewhere in the system (see Noble et al. 2014). However, the emphasis on a process of selection is correct, as are similar approaches found elsewhere (Dehaene and Changeux 2005; 2011).

The closest example of this emphasis on selection is in the work of Gerald Edelman (1978; Edelman et al. 2011) who as long ago as 1987 proposed the theory of neuronal group selection. And even earlier, Changeux and Danchin (1976) proposed the idea of selective stabilization of developing synapses. 
Furthermore, the relevant neural selection processes are extensively reviewed and extended by Ginsburg and Jablonka (2019). Chapter 3 of their book reviews previous work, while chapter 4 specifically tackles the tension (bridging) issue. Their work is a significant break with the long-standing tradition in the philosophy of mind to keep conceptual and empirical questions separate, a view stated most forcefully by (Bennett and Hacker 2003). We agree with Bennett and Hacker on the inadequacies of reductionism and the problems created by ascribing psychological attributes to parts of an animal that are intelligible only when ascribed to the animal as a whole. Nevertheless, we dissent from a hard separation of conceptual and empirical questions and argue that in a full understanding of conscious agency, there are contiguous processes. A hard separation makes it difficult to find processes by which the one can influence the other. This separation leads to a strange parallel dualism, the illusion of conscious agency, or the concept of a bit of the brain, the liaison brain, that can strangely communicate between mind and matter, the 'self' as separate from the 'body' (Popper and Eccles 1977), or a bit of the brain that sees the 'seeing' of the other.

None of this means that we cannot or should not consider conceptual issues in the abstract. Indeed, that is the essence of thinking and a fundamental ingredient of perception. Organisms, and certainly humans, can create and solve abstract problems. This paper is a testament to that. Animals can solve problems by both observation and imagination and from learning, as individuals and as social groups.

Nevertheless, what we measure and observe is, in no small degree, dependent on a conceptual framework. In biological processing, there is an interdependency between the conceptual and the empirical, although the relationship is asymmetrical. We can conceptualise with some degree of freedom from observation, but it is challenging to observe events without a conceptual framework. Arguably, thinking and anticipation are ingredients of perception.

This relationship between the conceptual and empirical brings us back to the central question addressed in this paper. If determinate molecular and other physical forces wholly and always determine the actions of organisms, then how could a rationalist explanation possibly be correct? The answer is that they do not. Organisms harness them in processes that maintain their integrity. Organisms create reasons, and abstract thinking by harnessing processes including stochasticity at the molecular, cellular, neural and social levels and are not abstract from them; they are the abstractors. By doing so, they anticipate the outcomes of their activities and that of others. They solve rational problems because they also create them. They are goal-directed. In this sense, they can generate and consider possible solutions to achieving their goals.

Even if we made what we consider to be an erroneous assumption that organisms were driven by genes to pass their genes on in a gene pool, they would have to overcome many problems in doing so. Yes, some solutions might be hard-wired, but many will require more flexible, creative solutions. As the chimpanzee learns, there may be more than one way to crack a nut (Luncz et al. 2012).

What we have shown in this article is that there is no difficulty from an empirical science viewpoint in envisaging how organisms could achieve conformity to rational actions through the process of harnessing stochasticity. Harnessing is a necessary causal process, as is evident also from its role in guiding evolution (Noble 2017; Noble and Noble 2017). Functional boundaries between organisational levels mean causation up and down are necessarily different (Noble et al. 2019), but they do not compete for primacy. They mesh together and are both enabling and creative. In setting boundaries, downward causation can be viewed more like a context, setting constraints, purpose and goals. It is then not too difficult to view reasons, ethics, laws and customs operating in 
this way. They are socio-biological processes influencing predisposition states in the organism. Thinking that we need to solve how upward and downward causation 'compete' with each other is a mistake. They mesh. Reasons are not incidental or merely epiphenomenal; organisms create them as contextual logic. Thus, reasons form the contextual influences within which action occurs. As we will make clear in Sect. 7 (Can reasons and values be causes?), this does not mean or guarantee that the decision will fully conform to any particular rationalisation. It is the taking of the decision in the context of seeking conformity to rationality that can be influential.

Living organisms are therefore capable of at least partially reconciling logical (reasons) and physical processes. They do this by reconciling different forms of causality. Causation across the boundaries between different levels is necessarily various (Noble et al. 2019). With the macro-level contextual constraints of events at the micro-level, there is no causal closure. The process is ongoing and reiterative with an ebb and flow of predisposition states influencing decisions by the organism. This openness, or lack of causal closure, is a point that seems to be missing in the accounts of philosophers like Jaegwon Kim (2000).

\section{Relevant Recent Philosophical Work}

The issue of free will has been a major one in philosophy for centuries (Kenny 2006, Ch. 7, 212-245; 2007, Ch. 8, 192-219) and the debate is still very much alive, as is clear from our earlier references to Jaegwon Kim's work.

In the introduction, we noted the relevant work of Karl Popper. We can now explain why we referred to close parallels with the proposition in this paper.

In his 1986 lecture to The Royal Society, Popper distinguished between what he called "active" and passive" Darwinism. By "passive" he meant a theory of evolution that attributes all change to natural selection, which is a passive filter for degrees of fitness for survival. This idea is also a central tenet of neo-Darwinism (The Modern Synthesis). In contrast, by "active" he meant the directionality that organisms create as agents, and which was first identified by Charles Darwin through his work on sexual selection-through an active choice of mates for reproduction (Darwin 1871).

The propositions in the current article contribute to understanding why Darwin was right to distinguish between natural selection and what he called artificial selectionthe active, or purposeful selection of varieties of species by human agency. The distinction is valid of course only if we believe that agents exist and that we can identify them. If active agency exists, then organisms are not passive prisoners of events. They can act to shape them.

Amongst modern philosophers, our proposals on the processes by which organisms make choices are closest to those of Christian List (List and Menzies 2009; List 2014, 2019).

List bases his arguments for free will on three propositions:

1. The fact that, for any macro-level situation, there will be innumerable micro-level states that correspond to the same macro-level state (List 2019, Fig. 2, 94).

2. From this, he shows that the high-level state can branch (in making a choice) while any given lower-level state does not and (in a deterministic world) cannot branch. 
3. From these statements, he shows that all three of his criteria for free-will are then satisfied. These are intentional agency, the existence of alternative possibilities, and causal efficacy.

We believe that our work provides strong support for (1).

Nevertheless, we are not convinced that, on its own, (2) would answer most micro-level determinists since they could still argue that in any given case only one of the many possible lower level instantiations occurs. Which one occurs can be described by pure biochemistry. Thus, they would argue that since on any given occasion, only one of them occurs then the particular high-level state cannot branch either. One way of viewing our contribution to the debate is to show that it is not necessary to assume that only one lower-level state occurs in any given timeline. Thus events at the macro-level harness those at a micro-level in generating possible alternative actions. Our case is based precisely on the organism's ability to anticipate many options simultaneously. It is the subsequent selection of one or more of these that is the choice process. The organism is then able to apply an ongoing creative logic to the choices of action, which in turn continuously moulds or modifies micro-level processes. Thus, the macro-level choices influence the biochemistry.

In terms of List's timeline diagrams for macro- and micro-levels (List 2019, 94), we are therefore arguing that the organism anticipates many of the lower-level timelines (whether consciously or not) when making choices. Rationality can enter into that choice, but that does not necessarily mean that the organism chooses the most rational that might be possible. Organisms do not behave as rational calculators. However, they are influenced by rational considerations, particularly in choices that anticipate the actions of other organisms or the outcome of their actions. These influences have causal efficacy because they establish an ebb and flow of predisposition states. Thus, anticipation is a crucial ingredient of all perception and all choices. Senses are not merely passive receivers. Our nervous systems anticipate change. Many illusions may result from tricks on the brain, resulting from this anticipation. We might better consider thought as integral to our senses rather than being nebulously superimposed upon them. There is then less difficulty in understanding how our thoughts can influence our behaviour. Thinking is a continuous process in the ebbing and flowing of the biological anticipatory process. Humans can do something remarkable with this, which is to use language better, convey abstract ideas to others and to express it culturally in art and literature, and this can alter our perceptions transgenerationally and the choices we might make. Ideas generated socially can alter our dispositional states. We have agency at both the organism and social level. We are free agents to the extent that we can run these processes freely to generate ideas on which we may act, although our capacity to do so is constrained. Learning and training can enhance our capacities.

By adding the harnessing of stochasticity as an active ingredient to List's set of propositions, it enables many micro-level states to be available to an organism in making choices. The argument for "free will", or as we would have it "free agency", becomes more tenable, and all the consequences of List's third proposition then follow.

\section{Can Reasons and Values Be Causes?}

The question of whether reasons, values, and other social/ethical factors can be regarded as causes is a contested one. Philosophers like, for example, Anthony Kenny argue that reasons cannot be causes: "reasons are not causes, and the relationship between reason and 
action is quite different from that between cause and effect." (Kenny 1992, 143). "This is because rules of practical inference are defeasible, whereas causal laws are not." "That is to say a conclusion which may be a reasonable one from a given set of premises may cease to be a reasonable one when further premises are added" (Kenny 1992, 145).

We agree that the rules of practical inference are defeasible. That is a necessary logical fact and not in question. Moreover, we have insisted throughout this article that actions that can be defended as reasonable are usually so defended in retrospect. As we wrote earlier "Organisms do not behave as rational calculators." We have also noted that, in everyday social interactions, and legal contexts, we do sometimes change the reasons we may acknowledge as to why we acted in the way we did. That also is not in question.

A choice of action may be its match to the plausibility of conforming to a rational construct. It is not caused in the way that micro-level events are caused by the physical dynamics of how material bodies interact, nor that it should follow the rules of practical inference. They are causal in the sense that they establish or alter states of disposition or be understood to work. Thus, the chimpanzee who has learned to crack nuts with stones will find a suitably shaped stone, which it may keep for future use if it is a useful utility for achieving its objective. We may consider the properties of a 'good' stone, such as its weight, shape and size, and how best to use it to good effect. Some chimpanzees will try different approaches; abandoning some stones on finding a "better."

What the process of choice through the harnessing of stochasticity requires is that organisms should be influenced (constrained) by considerations of rationality. Such constraint need not even be specific, or even justified, in particular cases. Furthermore, we explicitly include the fact that, often enough, in critically urgent circumstances, organisms simply do not have the time to arrive at the best response. Many actions in such cases are "on impulse". Organisms are also free to do that and will do so in crucial fight or flight situations. Being rational does not mean slavishly following a set of logic, just as we may not follow what we see or hear, touch or smell.

In these respects, the nature of the influence involved is comparable to that used by the immune system. A precisely exact fit of an antibody to the invading antigen is not necessary. What matters is that the fit is 'good enough'. If the key fits the lock, it does not have to be the perfect key.

Thus, we accept Kenny's point that reasons do not determine actions in the way that the dynamics of molecular interactions perform their role in causation. The process of harnessing stochasticity will always be, even at best, a 'good enough' response, not a logically calculated one. It involves a continuous appraisal and reappraisal of the solution and the problem.

As a specific example, consider the writing of this article. It took many iterations to arrive at the text. In highly deliberative actions, the outcome is a continuous striving towards a goal, in this case, to be as clear and logical as we can be. The logic we strove for was always defeasible. However, the striving towards that goal counts as a cause under the heading of "any factors whose alteration would result in changes in behaviour" (see Sect. 3. Causation). No specific (and defeasible) logical statement acted as the cause, but the general context of expressing our ideas logically was causal.

\section{Future Work}

Our article provides leads to future research, scientific and philosophical. 
a. Immune System The use we have made of the immune system as a model for the process of harnessing stochasticity is firm at the molecular level, but is far from being adequately investigated at the level of the overall control processes. How exactly do cells receiving signals at their surface communicate with such pinpoint accuracy to their genomes? Cellular communication mechanisms from surface to nucleus are now known in other physiological contexts (Ma et al. 2014; Kar et al. 2016), but we need to know much more about the integrative physiology of immune responses. This further understanding could provide valuable insights into other integrative biological processes employing stochasticity functionally.

b. Nervous System Further work on harnessing stochasticity in labile neural networks would provide insight into the processes of creative decision making. For example, this might involve control of synaptic function in labile switching of neuronal circuits underlying ideation.

c. Philosophy As our Sect. 6 shows, there are already ways in which the idea of harnessing stochasticity could link up with recent work on the philosophy of free agency. We anticipate that our article may open the way to future cooperation between scientists and philosophers in this field.

\section{Conclusions}

The conditions for the existence of what we call agency in this article include the following three scientific factors:

\subsection{The Openness of Organisms}

They are not and cannot be closed systems. All organisation levels nest within others. Once that is accepted, then a constraint of micro-level processes can occur through determining the initial and boundary conditions. Those constraints do not compete with the dynamics of the micro-level. The two forms of causation mesh together in achieving goal-directed behaviour.

\subsection{The Existence of Functional Boundaries, Both Between Different Levels of Organisation and Between Organisms and Their Environments}

Boundaries are the locations at which downward causes (constraint of micro-level elements by the macro-level organisation) exert their effects. The social boundary is, in some respects, similar to how a human agent interacts with a computer. On its own, the computer is a determinate mechanical system. Through its interaction across the human-machine boundary, it becomes constrained to instantiate the logic imposed by the human agency in writing programs and data.

\subsection{The Harnessing of Stochasticity, at Various Levels, Molecular, Cellular, Systems (e.g. Nervous System) and Social}

Thus, chance is not simply experienced; but used continuously in creatively guided behaviour. Merely incorporating random number generators into an otherwise determinate AI 
system does not capture this process. Harnessing of stochasticity can be either unconscious (immune system) or conscious (organisms with neural processes), even though the precise processes are different.

These conditions are interdependent. Openness automatically leads to the boundary between the organism and its environment. Harnessing stochasticity involves constraint both of micro-level chance events and socially-generated macro-level chance occurrences.

It will also be clear from this article that we regard organisms that have agency as actively anticipating their physical and social environments. Perception is an activity, not just a passive 'camera' observing the world.

But is the agency, according to these interpretations, really free? Yes, it is free of purely micro-level determination, thus giving it a degree of freedom, but it is still "determined" by the outcome of the meshing of macro-level constraints, including socially-determined constraints, with micro-level dynamics.

We, as humans, thinking we have what we call free will, would have no difficulty with someone saying of our reasons that they are what could be said to have determined what we did. That is how we answer questions such as "why did you do that?" So, in that sense, organisms with free will are determined, but by very complex logical, moral and other social factors meshing with the physical limitations on what organisms can do. Being open systems allows degrees of freedom in choice. Purpose and choice are determinate. It does not require a 'will' strangely posited such that it is 'free' from the material constraints. It is organisationally creative, harnessing the uncertainty of those constraints. Thus, we wrote this paper with the intention that the reader considers this point. It might bring about dispositional changes in the reader's brain that alter perception. Our rational actions are, in this sense, determined, but we determined them as purposeful agents.

In conclusion, the agency of organisms is powerful, not an illusion, and it is possible to reconcile the micro- and macro-level accounts of behaviour. Agency also endows organisms with directionality, i.e. intentional, forward-looking action. Agency gives an arrow to the interpretation of behaviour in much the same way as thermodynamics gives an arrow to time. Both do so through necessary constraints.

Acknowledgements We acknowledge valuable criticism of versions of this article from Anthony Kenny, George Ellis, Simona Ginsburg, and Eva Jablonka. We also thank the two anonymous referees who made valuable suggestions for revisions of this paper. Of course the errors that remain are our responsibility. Denis Noble is a member of the Project "Foundations of Value and Values" at the Said Business School of the University of Oxford and acknowledges valuable input from the members of that group. We declare no conflicts of interest.

Open Access This article is licensed under a Creative Commons Attribution 4.0 International License, which permits use, sharing, adaptation, distribution and reproduction in any medium or format, as long as you give appropriate credit to the original author(s) and the source, provide a link to the Creative Commons licence, and indicate if changes were made. The images or other third party material in this article are included in the article's Creative Commons licence, unless indicated otherwise in a credit line to the material. If material is not included in the article's Creative Commons licence and your intended use is not permitted by statutory regulation or exceeds the permitted use, you will need to obtain permission directly from the copyright holder. To view a copy of this licence, visit http://creativecommons.org/licenses/by/4.0/.

\section{References}

Atmanspacher, H., \& Rotter, S. (2011). On determinacy or its absence in the brain. In R. Swinburne (Ed.), Freewill and modern science. London: British Academy. 
Bathgate, K. E., Bagley, J. R., Jo, E., Talmadge, R. J., Tobias, I. S., Brown, L. E., et al. (2018). Muscle health and performance in monozygotic twins with 30 years of discordant exercise habits. European Journal of Applied Physiology. https://doi.org/10.1007/s00421-018-3943-7.

Bennett, M. R., \& Hacker, P. M. S. (2003). Philosophical foundations of neuroscience. Oxford: Blackwell Publishing.

Boyle, E. A. L., Li, Y. I., \& Pritchard, J. K. (2017). An expanded view of complex traits: from polygenic to omnigenic. Cell, 169, 1177-1186.

Brembs, B., \& Heisenberg, M. (2018). Der Zufall als kreatives Element in Gehirn und Verhalten. In U. Herkenrath (Ed.), Zufall in der belebten Natur. Hennef: Verlag Roman Kovar.

Bronfman, Z. Z., Ginsburg, S., \& Jablonka, E. (2016). The transition to minimal consciousness through the evolution of associative learning. Frontiers in Psychology, 7, 1954.

Burns, B. D. (1968). The uncertain nervous system. London: Arnold.

Byrne, R. W., Cartmill, E., Genty, E., Graham, K. E., Hobaiter, C., \& Tanner, J. (2017). Great ape gestures: intentional communication with a rich set of innate signals. Animal Cognition, 20, 755-769.

Capra, F., \& Luisi, P. L. (2014). The systems view of life: A unifying vision. Cambridge, UK: Cambridge University Press. https://doi.org/10.1017/CBO9780511895555.

Changeux, J.-P., \& Danchin, E. (1976). Selective stabilization of developing synapses as a machanism for the specification of neuronal networks. Nature, 264, 705-712.

Coyne, J. A. (2014). What scientific idea is ready for retirement? https://www.edge.org/response-detai 1/25381. Retrieved 12 October 2020.

Crick, F. H. C., \& Koch, C. (2003). A framework for consciousness. Nature Neuroscience, 6, 119-126.

Darwin, C. (1871). The descent of man, and selection in relation to sex. London: John Murray.

Dehaene, S., \& Changeux, J.-P. (2005). Ongoing spontaneous activity controls access to consciousness: A neuronal model for inattentional blindness. PLoS Biology, 3, e141.

Dehaene, S., \& Changeux, J.-P. (2011). Experimental and theoretical approaches to conscious processing. Neuron, 70, 200-227.

Del Santo, F., \& Gisin, N. (2019). Physics without Determinism: Alternative interpretations of classical physics. Physical Review A, 100, 062107.

Edelman, G. M. (1978). Neural Darwinism: the theory of neuronal group selection. New York: Basic Books.

Edelman, G. M., Gally, J. A., \& Baars, B. J. (2011). Biology of consciousness. Frontiers in Psychology. https://doi.org/10.3389/fpsyg.2011.00004.

Ellis, G. F. R. (2016). How can physics underlie the mind?. Berlin: Springer.

Epstein, D. (2014). The sports gene: Talent, practice and truth about success. Hyderabad: Yellow Jersey.

Foster, Russell, \& Kreitzman, Leon. (2004). Rhythms of life. London: Profile Books.

Ginsburg, S., \& Jablonka, E. (2019). The evolution of the sensitive soul. Cambridge: MIT Press.

Graham, K. E., Furuichi, T., \& Byrne, R. W. (2017). The gestural repertoire of the wild bonobo (Pan paniscus): A mutually understood communication system. Animal Cognition, 20, 171-177.

Graham, K. E., Hobaiter, C., Ounsley, J., Furuichi, T., \& Byrne, R. W. (2018). Bonobo and chimpanzee gestures overlap extensively in meaning. PLOS Biology. https://doi.org/10.1371/journal.pbio.2004825.

Heisenberg, M. (2009). Is free will an illusion? Nature, 459, 164-165.

Hille, B. (1992). Ionic channels of excitable membranes. Sunderland, Mass: Sinauer Associates Inc.

Hillenmeyer, M. E., Fung, E., Wildenhain, J., Pierce, S. E., Hoon, S., Lee, W., et al. (2008). The chemical genomic portrait of yeast: Uncovering a phenotype for all genes. Science, 320, 362-365.

Hobsiter, C., \& Byrne, R. W. (2014). The meanings of chimpanzee gestures. Current Biology, 24, 1-5.

Hoffmann, P. M. (2012). Life's Ratchet: How molecular machines extract order from chaos. New York: Basic Books.

Kar, P., Mirams, G. R., Christian, H. C., \& Parekh, A. B. (2016). Control of NFAT isoform activation and NFAT-dependent gene expression through two coincident and spatially segregated intracellular $\mathrm{Ca}^{2+}$ signals. Molecular Cell, 64, 746-759.

Kenny, A. J. P. (1992). The metaphysics of mind. Oxford: Oxford University Press.

Kenny, A. J. P. (2006). A new history of western philosophy. III The rise of modern philosophy. Oxford: Oxford University Press.

Kenny, A. J. P. (2007). A new history of western philosophy. IV philosophy in the modern world. Oxford: Oxford University Press.

Kim, Jaegwon. (2000). Mind in a physical world. Cambridge, Mass: MIT Press.

Koch, C. (2012). The quest for consciousness-confessions of a romantic reductionist. Cambridge: MIT Press.

Lim, D. (2008). Review of Murphy N \& Brown, W.S. 2007. Did My neurons make me do it? Philosophical and neurobiological perspectives on moral responsibility and free will. Zygon, 43, 748-753. 
List, C. (2014). Free will, determinism, and the possibility of doing otherwise. Nous, 48, 156-178.

List, C. (2019). Why free will is real. Harvard: Cambridge Mass.

List, C., \& Menzies, P. (2009). Non-reductive physicalism and the limits of exclusion. Journal of Philosophy, 106, 475-502.

Lucas, J.R. 1970. The freedom of the will (Oxford University Press).

Luncz, L. V., Mundry, R., \& Boesch, C. (2012). Evidence for cultural differences between neighboring chimpanzee communities. Current Anthropology, 22, 922-926.

Ma, H., Groth, R. D., Cohen, S. M., Emery, J. F., Li, B., Hoedt, E., et al. (2014). $\gamma \mathrm{CaMKII}$ shuttles $\mathrm{Ca}^{2+} /$ $\mathrm{CaM}$ to the nucleus to trigger CREB phosphorylation and gene expression. Cell, 159, 281-294.

Midgley, M. (2014). Are you an illusion?. London: Routledge.

Murphy, N., \& Brown, W. S. (2007). Did my neurons make me do it? Philosophical and neurobiological perspectives on moral responsibility and free will. Oxford: Oxford University Press.

Niemann, H.-J. (2014). Karl Popper and the two new secrets of life. Tuebingen: Mohr Siebeck.

Noble, D. (2012). A theory of biological relativity: No privileged level of causation. Interface Focus, 2, 55-64.

Noble, D. (2016). Dance to the tune of life. Biological relativity. Cambridge: Cambridge University Press.

Noble, D. (2017). Evolution viewed from physics, physiology and medicine. Interface Focus, 7, 20160159. https://doi.org/10.1098/rsfs.2016.0159.

Noble, D. (2018). Central dogma or central debate? Physiology. https://doi.org/10.1152/physiol.00017.2018.

Noble, R., \& Noble, D. (2017). Was the watchmaker blind? Or was she one-eyed? Biology, 6, 47. https://doi. org/10.3390/biology6040047.

Noble, R., \& Noble, D. (2018). 'Harnessing stochasticity. How Organisms Make Choices', Chaos, 28, 106309. https://doi.org/10.1063/1.5039668.

Noble, R., \& Noble, D. (2019). Could artificial intelligence (AI) become a responsible agent: Atificial agency (AA)? RUSI Journal, 164, 130-135.

Noble, D., Noble, R., \& Schwaber, J. (2014). What is it to be conscious? In J. Smythies, V. S. Ramachandran, \& L. Edelstein (Eds.), The claustrum. Cambridge: Academic Press.

Noble, R., \& Riddell, J. S. (1989). Descending influences on the cutaneous receptive fields of postsynaptic dorsal column neurones in the cat. Journal of Physiology, 408, 167-183.

Noble, R., \& Short, A. D. (1989). Spatial spread of in-field afferent inhibition in the cat's spinocervical tract. Journal of Physiology, 413, 107-118.

Noble, R., Tasaki, K., Noble, P. J., \& Noble, D. (2019). Biological Relativity requires circular causality but not symmetry of causation: So, where, what and when are the boundaries? Frontiers in Physiology, 10, 827.

Odegard, V. H., \& Schatz, D. G. (2006). Targeting of somatic hypermutation. Nature Reviews Immunology, $8,573-583$.

Plomin, R. (2018). Blueprint: How DNA makes us who we are. Bristol: Allen Lane.

Popper, K. (1945). The open society and its enemies. London: Routledge.

Popper, K. R. (1972). Objective knowledge. An evolutionary approach. Oxford: Oxford University Press.

Popper, K. R. (1973). Indeterminism is not enough. Encounter, 40, 20-26.

Popper, K. R., \& Eccles, J. C. (1977). The self and its brain. New York: Springer.

Sakai, T., Tamura, T., Kitamoto, T., \& Kidokoro, Y. (2004). A clock gene, period, plays a key role in long-term memory formation in Drosophila. Proceedings of the National Academy of Sciences, 101, $16058-16063$.

Saribasak, H., \& Gearhart, P. (2012). Does DNA repair occur during so0matic hypermutation? Seminars in Immunology, 24, 287-292. https://doi.org/10.1016/j.smim.2012.05.002.

Shapiro, James A. (2011). Evolution: A view from the 21st century. Upper Saddle River, NJ: Pearson Education Inc.

Smith, J. M. (1979). Letter. The New Scientist, 14 June 1979, 213.

Smith, J. M. (1998). The units of selection. In The limits of reductionism in science. Wiley.

Smith, Maynard, \& John, (1964). Group and kin selection. Nature, 201, 1145-1147.

Tchaptchet, A., Jin, W., \& Braun, H. A. (2015). Diversity and noise in neurodynamics across different functional levels. In R. Wang \& X. Pan (Eds.), Advances in cognitive neurodynamics. Singapore: Springer.

Vohs, K. D., \& Schooler, J. W. (2008). The value of believing in freewill, encouraging a belief in determinism increases cheating. Psychological Science, 19, 49-54.

Whiten, A. (2017). A second inheritance system: The extension of biology through culture. Interface Focus. https://doi.org/10.1098/rsfs.2016.0142.

Publisher's Note Springer Nature remains neutral with regard to jurisdictional claims in published maps and institutional affiliations. 